\section{Commentary: Esophageal squamous cell carcinoma: A disease requiring more research}

\author{
Ke-Neng Chen, MD, PhD
}

The prospective study "Does FDG-PET/CT Have a Role in Cervical Nodal Staging for Esophageal Squamous Cell Carcinoma?" conducted by $\mathrm{Li}$ and colleagues ${ }^{1}$ investigated the diagnostic value of positron emission tomography/ computed tomography (PET/CT) for cervical lymph node metastasis (LNM) of esophageal squamous cell carcinoma (ESCC). The study evaluated the sensitivity, specificity, positive predictive value, negative predictive value, and accuracy of PET/CT based on postoperative (3-field lymph node dissection [3f]) pathology, and a comparison between $\mathrm{PET} / \mathrm{CT}$ and cervical ultrasound was also performed. The results showed that there was no significant difference in sensitivity, specificity, positive predictive value, negative predictive value, or accuracy between PET/CT and ultrasound in terms of LNM diagnosis. The authors concluded that PET/CT was not useful in the diagnosis of LNM.

There are several merits in this study: (1) It is a prospective and multicenter study; (2) both PET/CT and ultrasound were performed preoperatively; and (3) most importantly, patients were without induction therapy, and $3 \mathrm{f}$ were routinely performed, so that direct comparison of preoperative $\mathrm{N}$-status and postoperative LNM could be done. However, the diagnosis and treatment in this study need to be clarified, which, on the one hand, reflects that the existing guidelines are not widely accepted in the current clinical practice and, on the other hand, it fully reveals the complexity of ESCC itself. Therefore, it is reasonable to say that ESCC is a disease requiring more research.

For a long time, the treatment of ESCC has been surgery alone or surgery plus postoperative adjuvant treatment, with unsatisfactory effects. Therefore, clinicians have been exploring more appropriate perioperative treatment that can improve patients' outcomes. After the failure of

From Thoracic Surgery, Peking University Cancer Hospital, Beijing, China.

Disclosures: Author has nothing to disclose with regard to commercial support.

Received for publication Nov 26, 2019; revisions received Nov 26, 2019; accepted for publication Nov 27, 2019; available ahead of print Dec 14, 2019.

Address for reprints: Ke-Neng Chen, MD, PhD, No. 52, Fucheng Rd, Haidian Dis-

trict, Beijing, China, 100142 (E-mail: chenkeneng@bjmu.edu.cn).

J Thorac Cardiovasc Surg 2020;160:553-4

0022-5223/ $\$ 36.00$

Copyright (c) 2019 by The American Association for Thoracic Surgery

https://doi.org/10.1016/j.jtcvs.2019.11.112

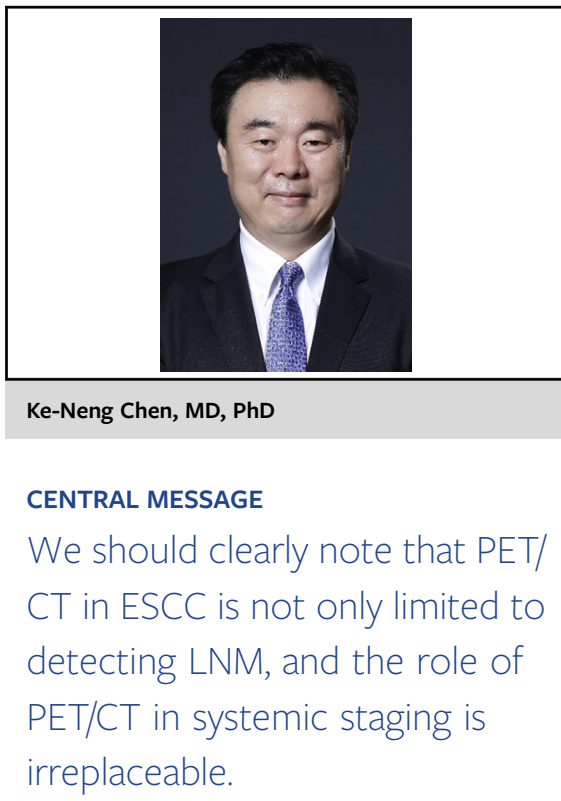

preoperative radiotherapy and the controversy of preoperative chemotherapy, CROSS (Neoadjuvant chemoradiotherapy plus surgery versus surgery alone for oesophageal or junctional cancer) ${ }^{2}$ and $5010^{3}$ established the optimal position of concurrent chemoradiotherapy in the neoadjuvant setting of locally advanced ESCC. Our recent survey in China also indicated that $80 \%$ of physicians accept induction strategies. What needs further exploration is the choice among various preoperative treatments, such as chemotherapy alone, chemoradiotherapy, or other treatment combinations.

The status of LNM is important in determining the treatment strategy for ESCC. Worldwide, the staging systems of ESCC used in clinical practice are the American Joint Committee on Cancer (AJCC) and the Japan Esophageal Society (JES). ${ }^{4,5}$ The definitions of the $\mathrm{T}$ and $\mathrm{M}$ in the 2 systems are basically the same. The major differences between the 2 systems are the definitions of LNM. In JES, the spatial relations of LNM to the primary tumors are more emphasized. Therefore, all LNM along the esophagus, including supraclavicular LNM, is defined as regional LNM, which is classified as $\mathrm{N} 1$ to $\mathrm{N} 3$ according to the distances to tumor and could reflect patients' survival after resection. Therefore, the importance of surgical resection is emphasized. On the other hand, AJCC focuses on gastroesophageal junction adenocarcinoma, $\mathrm{N}$ staging is based on numbers of LNM, and "cervical" is defined as distant metastasis, implying the extent of esophagectomy without the dissection of cervical LNM. The rationale behind $3 \mathrm{f}$ is that surgeons believe 
that it could cure the disease, and other potential reasons include LNM could occur in any part along the esophagus for ESCC of various locations. Second, the commonest recurrence of ESCC postoperatively is LNM. Third, the curative effect of systemic therapy for ESCC has been limited in the past. Finally, it could provide more accurate staging.

Nevertheless, the essence of the 2 staging systems for $\mathrm{N}$ category is basically same in clinical significance. Udagawa and colleagues ${ }^{6}$ retrospectively analyzed 806 cases that underwent $3 \mathrm{f}$ respectively and pointed out that both systems (AJCC and JES) had similar prognostic value: the survival curves of the patients by the 2 staging systems were fairly overlapped. That is to say, $3 \mathrm{f}$ could not directly improve patients' long-term survival, indicating that the diagnostic significance is superior to its treatment significance.

Moreover, we should note that PET/CT in ESCC is not only limited in detecting LNM. In other words, the determination of cervical LNM is not the major objective of PET/ CT. First, the theoretical basis of PET/CT is to screen the whole body. Our previous study found that PET/CT could identify $8 \%$ more metastases that were undetectable otherwise by traditional imaging methods and thus avoided unnecessary surgery. ${ }^{7}$ From this perspective, PET/CT is irreplaceable in clinical practice. Second, due to its ability to reflect metabolic levels, PET/CT is also an important tool to evaluate the activities of tumors as well as efficacy of induction therapy. Therefore, although the value of PET/CT in the evaluation of LNM is relatively low, the importance of PET/CT in treatment of ESCC should not be underscored. Due to the deep location of esophagus, it is not easy to confirm LNM. Therefore, the preoperative evaluation is often based on clinical examinations, including CT, ultrasound, magnetic resonance imaging, and PET/CT. However, each examination has its own limitations, and no one can replace the other. Therefore, the diagnoses of LNM require multiple dimensions to render the most accurate staging as possible. In this regard, PET/CT is undoubtedly a valuable examination.

To sum up, first, we should clearly recognize the irreplaceable role of PET/CT in systemic staging. Second, multiple methods are needed in the evaluation of LNM to maximize the diagnostic accuracy. Third, like other solid malignancies, ESCC should be regarded as systemic disease, and no matter "how complete" the local treatment is, induction systemic treatment for locally advanced ESCC is undoubtedly necessary.

\section{References}

1. Li B, Li N, Liu S, Li Y, Qian B, Zhang Y, et al. Does [18F] fluorodeoxyglucosepositron emission tomography/computed tomography have a role in cervical nodal staging for esophageal squamous cell carcinoma? J Thorac Cardiovasc Surg. 2020;160:544-50.

2. Shapiro J, Van Lanschot JJB, Hulshof MCCM, van Hagen P, van Berge Henegouwen MI, Wijnhoven BPL, et al. Neoadjuvant chemoradiotherapy plus surgery versus surgery alone for oesophageal or junctional cancer (CROSS): long-term results of a randomised controlled trial. Lancet Oncol. 2015;16:1090-8.

3. Yang H, Liu H, Chen Y, Zhu C, Fang W, Yu Z, et al. Neoadjuvant chemoradiotherapy followed by surgery versus surgery alone for locally advanced squamous cell carcinoma of the esophagus (NEOCRTEC5010): a phase III multicenter, randomized, open-label clinical trial. J Clin Oncol. 2018;36:2796-803.

4. Rice TW, Ishwaran H, Ferguson MK, Blackstone EH, Goldstraw P. Cancer of the esophagus and esophagogastric junction: an eighth edition staging primer. $J$ Thorac Oncol. 2017;12:36-42.

5. Japan Esophageal Society. Japanese Classification of Esophageal Cancer, 11th edition: part I. Esophagus. 2017;14:1-36.

6. Udagawa H, Ueno M, Shinohara H, Haruta S, Kaida S, Nakagawa M, et al. The importance of grouping of lymph node stations and rationale of three-field lymphoadenectomy for thoracic esophageal cancer. J Surg Oncol. 2012;106: 742-7.

7. Wang F, Shen LY, Ma SH, Li N, Yang Z, Chen KN. Advantages of positron emission tomography-computed tomography in esophageal squamous cell carcinoma. Dis Esophagus. 2013;26:832-7. 